\title{
Searching for star formation beyond reionization
}

\section{Citation}

Barton, Elizabeth J., Romeel Dav, John-David T. Smith, Casey Papovich, Lars Hernquist, and Volker Springel. 2004. "Searching for Star Formation beyond Reionization." The Astrophysical Journal 604 (1): L1-4. https://doi.org/10.1086/383489.

\section{Permanent link}

http://nrs.harvard.edu/urn-3:HUL.InstRepos:41381822

\section{Terms of Use}

This article was downloaded from Harvard University's DASH repository, and is made available under the terms and conditions applicable to Other Posted Material, as set forth at http:// nrs.harvard.edu/urn-3:HUL.InstRepos:dash.current.terms-of-use\#LAA

\section{Share Your Story}

The Harvard community has made this article openly available.

Please share how this access benefits you. Submit a story.

\section{Accessibility}




\title{
SEARCHING FOR STAR FORMATION BEYOND REIONIZATION
}

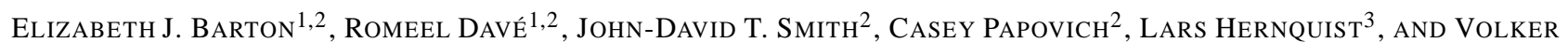 \\ SPRINGEL $^{4}$ \\ submitted to ApJ Letters
}

\begin{abstract}
The goal of searching back in cosmic time to find star formation during the epoch of reionization will soon be within reach. We assess the detectability of high-redshift galaxies by combining cosmological hydrodynamic simulations of galaxy formation, stellar evolution models appropriate for the first generations of stars, and estimates of the efficiency for Ly $\alpha$ to escape from forming galaxies into the intergalactic medium. Our simulated observations show that Ly $\alpha$ emission at $z \sim 8$ may be observable in the near-infrared with 8-meter class telescopes and present-day technology. Not only is the detection of early star-forming objects vital to understanding the underlying cause of the reionization of the universe, but the timely discovery of a $z>7$ star-forming population - or even an interesting upper limit on the emergent flux from these objects - will have implications for the design of the next generation of ground- and space-based facilities.
\end{abstract}

Subject headings: galaxies: evolution — galaxies: formation — galaxies: high-redshift

\section{INTRODUCTION}

Recent observations have significantly advanced our understanding of the reionization of the universe. Estimates of the Thomson optical depth from the Wilkinson $\mathrm{Mi}$ crowave Anisotropy Probe (WMAP) suggest that the reionization epoch began at redshifts $14 \lesssim z \lesssim 20$ (Bennett et al. 2003; Spergel et al. 2003) and Gunn-Peterson troughs in distant quasars indicate that reionization ended at $z \sim 6$ (Becker et al. 2001; Fan et al. 2002). However, the known population of quasars cannot produce sufficient ionizing radiation (Fan et al. 2001), so star-forming galaxies are most likely the dominant source of ionizing photons at redshifts $z>7$, a view supported by theoretical studies of reionization (e.g., Tinslev 1973; Sokasian et al. 2003a). The direct detection of these objects lies at the next frontier in the study of the evolution of the early universe.

Discoveries of star-forming objects are progressing to ever higher redshifts, with successful results from both broad-band color selection (e.g., Yan. Windhorst. \& Cohen 2003; Stanway, Bunker, \& McMahon 2003; Bunker et al. 2003; Dickinson et al. 2003) and Ly $\alpha$ emission line searches (e.g., Rhoads et al. 2003; Maier et al. 2003; Cuby et al. 2003). Although the number counts of $I$-band "dropouts" (at $5.5 \lesssim z \lesssim 6.5$ ) are still heavily debated, it is clear that a substantial population of galaxies with detectable continuum exists at these redshifts. The next step - discovering a population of objects before the end of reionization - lies behind a technological barrier: most of the light from $z>7$ objects falls in the near-infrared, where backgrounds provide significant challenges from the ground. High spectral resolution observations provide some relief; dispersing the light eliminates much of the background noise and allows the detection of line emission (e.g., Thompson, Diorgovski, \& Trauger 1995; Thompson. Mannucci. \& Beckwith 1996; Panagia. Stiavelli, \& Fall 2003).

At first glance, weak star formation and a neutral intergalactic medium (IGM) would appear to critically hamper the detection of $\operatorname{Ly} \alpha$ at high redshift. However, a top-heavy initial

\footnotetext{
${ }^{1}$ Hubble fellow

${ }^{2}$ University of Arizona, Steward Observatory, 933 N. Cherry Ave., Tucson, AZ 85721

${ }^{3}$ Harvard-Smithsonian Center for Astrophysics, 60 Garden Street, Cambridge, MA 02138

4 Max-Planck-Institut für Astrophysik, karl-Schwarzschild-Stra $\beta$ e 1, 85740 Garching bei München, Germany
}

stellar mass function (IMF) and low metallicity may boost the Ly $\alpha$ flux of the earliest stars (e.g.,Bromm. Kudritzki. \& Loeb 2001; Schaerer 2003). In addition, this emission may penetrate even a neutral IGM with reasonable efficiency (Haiman 2002; Santos 2003).

Taken together, the arguments above strongly motivate the search for Ly $\alpha$ emission at redshifts $z \geq 7$ using narrow-band imaging in the near-infrared. Here, we describe a search for Ly $\alpha$ in a narrow $J$-band "window" in the sky background, corresponding to $z \sim 8$.

\section{STAR-FORMING OBJECTS AT $Z \approx 8$}

\subsection{Hydrodynamical Simulations of Early Star Formation}

We examine the star formation rates in the cosmological simulations of Springel \& Hernquist (2003a). Employing a novel treatment of smoothed particle hydrodynamics and a multiphase description of star-forming gas to incorporate feedback and stellar winds (Springel \& Hernquist 2002, 2003b), Springel \& Hernquist (2003a) obtained a numerically converged prediction for the cosmic star formation rate (SFR) as a function of redshift that agrees with the observed star formation history at low redshift. ${ }^{5}$ Here, we focus on the $10 h^{-1}$ comoving Mpc Q5 simulation of a WMAPconcordant cosmology with $2 \times 324^{3}$ particles. The more luminous sources in the simulations have star formation rates in the range of $10^{-2} \leq \dot{\mathrm{M}} \lesssim 1 \mathrm{M}_{\odot} / \mathrm{yr}$. With typical dynamical masses of $10^{8}-10^{9} \mathrm{M}_{\odot}$ at $z=8$, their intrinsic circular velocities are generally $30-50 \mathrm{~km} \mathrm{~s}^{-1}$. The simulations predict an early rise in star formation activity at $z \geq 5$; at $z=8$, the star formation rate density is within a factor of $\sim 2$ of its peak, relatively independent of the details of the physics of star formation and feedback (Hernquist \& Springel 2003).

\subsection{Ly $\alpha$ Emission and Absorption: the IMF, the IGM, and the ISM}

The amount of Ly $\alpha$ that we can observe from young starforming objects depends on the IMF and metallicity in early galaxies, the amount of attenuation within the galaxy itself, and the amount of scattering in the intergalactic medium:

$$
\mathrm{F}_{\text {Ly } \alpha, \text { obs }}=\mathrm{e}^{-\tau_{\text {dust }}}\left(1-\mathrm{f}_{\text {esc }}\right) \mathrm{f}_{\text {IGM }} \mathrm{F}_{\text {Ly } \alpha, \text { em }}
$$

\footnotetext{
${ }^{5}$ We note an error in figure 12 of Springel \& Hernquist (2003a) in which the observational estimates of the SFR were plotted too high by a factor of $h^{-1}=1.4$. When corrected, the observed points are in better agreement with their theoretical estimates.
} 
where $\mathrm{F}_{\mathrm{Ly} \alpha, \mathrm{obs}}$ is the observable $\mathrm{Ly} \alpha$ flux per unit of star formation, $\tau_{\text {dust }}$ is the optical depth due to dust in the galaxy, $\mathrm{f}_{\text {esc }}$ is the fraction of ionizing photons that escape the starforming galaxy and thus create no Ly $\alpha$ photons at the source, and $f_{I G M}$ is the total fraction of Ly $\alpha$ photons that penetrate the intergalactic medium and reach us. $\mathrm{F}_{\mathrm{Ly} \alpha, \mathrm{em}}$ is the maximum, ionization-bounded flux of Ly $\alpha$ photons produced per unit of star formation; it depends on the IMF and metallicity. Recent models and the interpretation of high-redshift galaxies provide some constraints on these quantities.

The IMF: Theoretical models predict a top-heavy IMF for the first generation of stars formed in metal-free gas (Bromm. Coppi. \& Larson 1999, 2002; Abel. Bryan. \& Norman 2000). These stars can radiate more than an order of magnitude more ionizing photons per unit solar mass than a Salpeter IMF, resulting in much stronger Ly $\alpha$ and He II $(\lambda 1640)$ Bromm, Kudritzki, \& Loeb 2001; Schaerer 2003). However, only the initial generations of stars will be formed from completely pristine gas; thus, one major source of uncertainty is whether luminous bursts of star formation later in the process $(z=8)$ will be too enriched to exhibit extremely top-heavy IMFs.

The ISM: The factor $\mathrm{e}^{-\tau_{\text {dust }}}\left(1-\mathrm{f}_{\text {esc }}\right)$ is the fraction of emitted ionizing photons available for conversion to $\operatorname{Ly} \alpha$; it includes the absorption of ionizing and Ly $\alpha$ photons from dust near the newly forming stars. There are, at present, few direct observational constraints on this factor at any redshift. At $z \sim 3$, Pettini et al. (1998) estimate $\mathrm{e}^{-\tau_{\text {dust }}} \sim 0.16-0.4$. But the stellar populations at even higher redshift are probably younger, less chemically evolved, and hence less dusty. For the escape fraction, Steidel. Pettini. \& Adelberger (2001) combine $z \sim 3$ Lyman-break galaxy spectra to estimate an $a v$ erage $\mathrm{f}_{\mathrm{esc}} \gtrsim 0.07-0.1$.

The IGM: The discovery of Gunn-Peterson troughs in the spectra of quasars at $z \gtrsim 6$ indicate that the average neutral fraction of the intergalactic medium exceeds $x_{\mathrm{HI}} \sim 10^{-2}$ at $z \gtrsim 6$ (Becker et al. 2001; Fan et al. 2002). At face value, this result suggests that $\operatorname{Ly} \alpha$ emission from beyond this redshift will not penetrate the IGM. However, Haiman (2002) shows that a substantial fraction of the emitted $\operatorname{Ly} \alpha$ photons escape scattering by the IGM when a galaxy ionizes a local bubble in its immediate surroundings. Extending this idea using dynamical models of the IGM and galactic winds, Santos (2003) derives values of $\mathrm{f}_{\mathrm{IGM}}$ ranging from $\lesssim 0.002$ to $\sim 1$. This broad range illustrates the substantial uncertainties involved in estimating the expected Ly $\alpha$ flux. In general, penetration through the IGM improves for galaxies with lower redshifts, higher star-formation rates, and older (longer-duration) bursts of star formation, higher $\mathrm{f}_{\mathrm{esc}}$, outflows in which the centroid of Ly $\alpha$ is shifted redward, and with higher ionizing backgrounds in the universe. Because unabsorbed ionizing photons either create $\operatorname{Ly} \alpha$ in the source or escape to ionize the IGM, but not both, the total emergent Ly $\alpha$ decreases for both very high and very low escape fractions. The fiducial models of Santos (2003) show a broad peak in detectable $\mathrm{Ly} \alpha$ at $\mathrm{f}_{\text {esc }} \sim 0.1-0.8$.

The strongest constraints on the creation and transmission of Ly $\alpha$ come from the cosmic microwave background. The WMAP observation of the Thompson optical depth to reionization, $\tau_{e}=0.17 \pm 0.4$ (Kogut et al. 2003), constrains the parameters in Equation 1 By modeling the reionization process, Cen (2003a,b) and Sokasian et al. (2003b) find that a large Thomson optical depth requires most or all of the following at high redshift: a top-heavy IMF of extremely lowmetallicity stars, a high star formation efficiency in low-mass halos, $f_{\text {esc }} \gtrsim 0.3$, a positive tilt in the matter power spectrum, or an additional source of ionizing photons. Except for escape fractions extremely close to unity, these conditions are

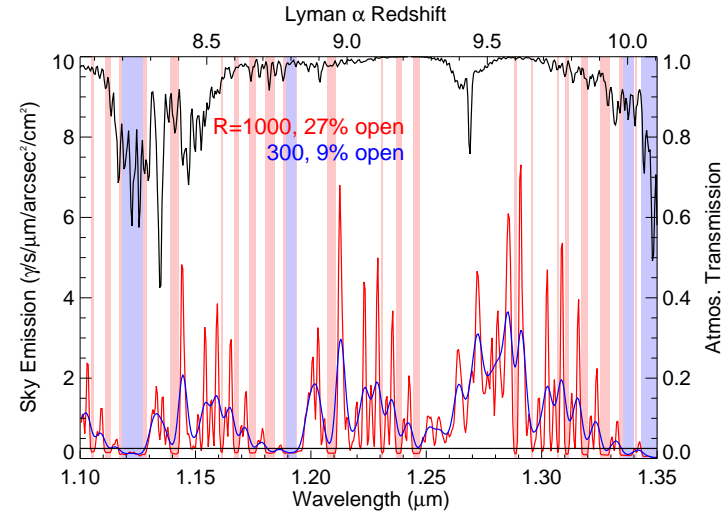

FIG. 1._ "Windows" in the $J$-band night sky spectrum. The black line indicates the transmission of the night sky (scale on right). We plot the night sky spectrum at two resolutions $(R=1000$, red line; $R=300$, blue line $)$ to indicate regions below $1 / 4$ of the mean background (blue shading for moderate resolution, red shading for high resolution). At moderate resolution, only a small number of windows are available.

all conducive to observing $z>7$ galaxies in Ly $\alpha$.

Although much uncertainty surrounds the search for Ly $\alpha$ in $z>7$ galaxies, we argue that the distinct possibility of success motivates an attempt with present-day technology. As an illustration, we consider two specific scenarios that are both detectable in Ly $\alpha$ by design. They differ by a factor of $\sim 3.4$ in the flux of observable Ly $\alpha$ photons. They are:

1. Plausible: An IMF rich in massive stars with a Salpeter slope, containing only stars in the range $50-$ $500 \mathrm{M}_{\odot}$, with a metallicity of $\mathrm{Z}_{\odot}=0$, an escape fraction of $\mathrm{f}_{\text {esc }}=0.1, \tau_{\text {dust }}=0$, and $\mathrm{f}_{\mathrm{IGM}}=0.25$, which is appropriate for a range of IGM wind models, yielding $\mathrm{F}_{\mathrm{Lv} \alpha \text {,obs }}=6.4 \times 10^{42} \mathrm{erg} \mathrm{s}^{-1} /\left[\mathrm{M}_{\odot} / \mathrm{yr}\right]$ of detectable Ly $\alpha$ (Schaerer 2003; Santos 2003).

2. Optimistic: An extremely top-heavy IMF composed of only 300-1000 M $\mathrm{M}_{\odot}$ zero-metallicity stars (Bromm. Kudritzki. \& Loeb 2001), $\tau_{\text {dust }}=0, \mathrm{f}_{\text {esc }}=$ 0.35 to maximize the observable Ly $\alpha$ emission based on the fiducial Santos (2003) models, and $\mathrm{f}_{\mathrm{IGM}}=1$, appropriate for an IGM model with some ionizing background in which strong galactic winds clear the Stromgren spheres around galaxies and shift Ly $\alpha$ toward the red (Santos 2003). This combination yields $\mathrm{F}_{\mathrm{Ly} \alpha, \text { obs }}=2.1 \times 10^{43} \mathrm{erg} \mathrm{s}^{-1} /\left[\mathrm{M}_{\odot} / \mathrm{yr}\right]$ of detectable Ly $\alpha$.

Both scenarios make assumptions that are relatively favorable for detecting Ly $\alpha$ emission, such as the presence of a top-heavy IMF in high-redshift galaxies. If regions of intense star formation are sufficiently chemically enriched by $z \sim 8$ to have a Salpeter IMF from $1-100 \mathrm{M}_{\odot}, \mathrm{F}_{\mathrm{Ly} \alpha, \mathrm{em}}$ is reduced by a factor of $4-12$, depending on metallicity. With $6.3 \times 10^{8}$ years of cosmic time available for star formation before $z=8$, delaying chemical enrichment sufficiently long may require suppressing star formation at early times, as would be the case if the power spectrum were reduced on small scales (e.g., Yoshida et al. 2003a b). In addition, inhomogeneous structure formation may lead to pockets of low-metallicity star formation even at late epochs (Scannapieco, Schneider, \& Ferrara 2003). Observationally, rest-frame equivalent widths of Ly $\alpha$ as late as $z \sim 5.7$ support the hypothesis that the IMF may be top-heavy at late epochs (e.g., Rhoads et al. 2003). More indirect arguments in favor of massive and/or low-metallicity early stars come from comparisons of the star formation history of the universe to the requirements for reionization (e.g., 


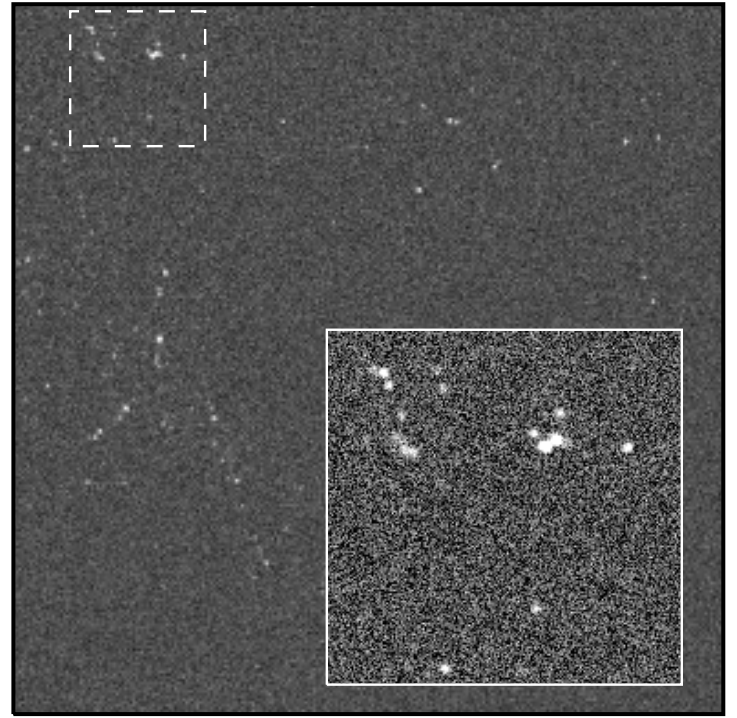

FIG. 2.- A realization of a 32-hour $2^{\prime} \times 2^{\prime}$ observation of the "optimistic" scenario with an 8-meter telescope. We simulate detection of the objects in the Springel \& Hernquist (2003a) Q5 $10 \mathrm{~h}^{-1}$ comoving Mpc model. The $R=125$ narrow-band filter we assume is actually $44 \%$ thicker in redshift depth than this simulated observation. There are 56 detectable sources in the field. We show the dotted inset enlarged for clarity.

Ferguson. Dickinson. \& Papovich 2002) and from fossil elemental abundances (Beaslev et al. 2003).

\section{IDENTIFYING AND MAPPING HIGH-REDSHIFT SOURCES}

A simple argument shows that for even a moderate $\operatorname{Ly} \alpha$ emitter, detecting Ly $\alpha$ from the ground at high spectral resolution is easier than detecting the continuum emission. For example, in the J-band window at $1.122 \mu m$ ( $\mathrm{Ly} \alpha$ at $\mathrm{z}=8.2$; see Sec. 3.1), assuming a Mauna Kea sky background and observations in which sky noise dominates, the signal-to-noise ratio of a suitably located $R=200$ observation of $\operatorname{Ly} \alpha$ becomes equal to or better than that of a broad-band continuum observation $(R=4)$ when the rest-frame $\mathrm{EW}(\mathrm{Ly} \alpha)_{\mathrm{em}} \gtrsim 27 \AA$. At $R=2000$, the threshold is $\mathrm{EW}(\operatorname{Ly} \alpha)_{\mathrm{em}} \gtrsim 8 \AA$. Observed restframe Ly $\alpha$ equivalent widths of $z=5.7$ galaxies exceed $150 \AA$ Rhoads et al. 2003) and starburst models predict equivalent widths far in excess of $100 \AA$ for most young starburst scenarios (Bromm. Kudritzki, \& Loeb 2001; Schaerer 2003). Thus, detection in Ly $\alpha$ is likely the most powerful way to identify and map very high-redshift star formation from the ground.

Observing spectral features between the strong atmospheric lines helps to minimize the background in the near-infrared. Although there are existing spectrographs with high enough resolution to observe between $\mathrm{OH}$ lines, the relatively low number density of detectable objects predicted by simulations argues for blind searches for Ly $\alpha$ emitters in a large, contiguous field ( $\gtrsim$ few $\square^{\prime}$ ). Because narrow-band filters with $R \gtrsim 200$ are not widely available, the most effective current strategy is to identify the broadest $\mathrm{OH}$-free windows in the night sky; Fig. 11illustrates some windows in the $J$-band, including a wide window at $z=8.2-8.3$ with $\sim 80 \%$ (variable) atmospheric transmission. ${ }^{6}$

\subsection{A Simulated Observation with an 8-meter Telescope}

We assume each star-forming particle in our simulation is a source of Ly $\alpha$ photons, converting star formation rate to $\mathrm{F}_{\mathrm{Ly} \alpha, \text { obs }}$ as described in $\$ 2.2$ For an $R=125$ narrow-band filter in the $z=8.227 \mathrm{~J}$-band window, 0 "' 35 seeing, a total sys-

\footnotetext{
${ }^{6}$ We use the Gemini sky spectrum from http://www.gemini.edu
}

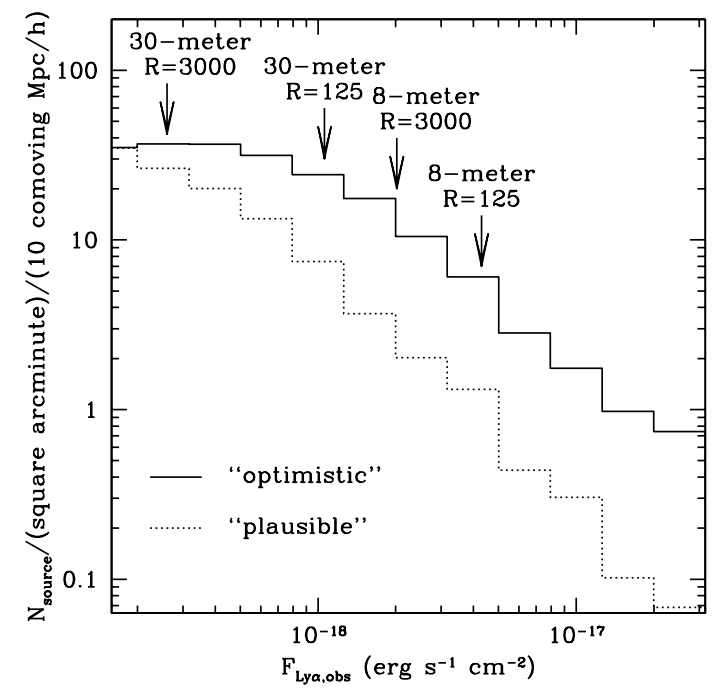

FIG. 3.- Ly $\alpha$ fluxes in the $z=8.227 \mathrm{~J}$-band window. We plot number counts per square arcminute in the Springel \& Hernquist [2003a) Q5 $10 \mathrm{~h}^{-1}$ comoving Mpc simulation, converting star formation rates to observable Ly $\alpha$ fluxes via the "plausible" and "optimistic" scenarios outlined above. The arrows indicate detection limits for 32-hour exposures with 8-meter and 30 -meter telescopes at moderate $(R=125)$ and high $(R=3000)$ resolutions.

tem throughput of $20 \%$, and the detector parameters appropriate for a modern detector (the NIRI detector on Gemini), we simulate 32-hour observations of Ly $\alpha$ for the "optimistic" and "plausible" scenarios. Fig. 2 shows a $2^{\prime} \times 2^{\prime}$ corner of the "optimistic" simulation; the thickness of the simulation is $44 \%$ less than the thickness of an $R=125$ observation, so the simulation actually underrepresents the number of sources expected in this scenario.

Allowing for the depth and angular size of the simulation, the volume probed by the entire Q5 model is 5.14 times larger than the volume of a single $R=125$ narrow-band filter observation with a $2^{\prime} \times 2^{\prime}$ detector. We analyze the simulated observations with the SExtractor software (Bertin \& Arnouts 1996). For the "optimistic" case, we find 176 sources in the entire simulation volume to a $\mathrm{S} / \mathrm{N}$ of $\gtrsim 3.4$. Although the segment in Fig. 2 2 is in a well-populated part of the simulation, with 56 detectable sources, the detectable objects do cover the volume well in the sense that any $2^{\prime} \times 2^{\prime}$ field would include observable galaxies. In the "plausible" simulation case, we detect 23 sources. The objects are clustered, but a $2^{\prime} \times 2^{\prime}$ observation would reveal no sources $\lesssim 20 \%$ of the time.

\subsection{Identifying Low-redshift Contaminants}

Although detecting galaxies via their Ly $\alpha$ emission is the primary challenge, separating the sources from possible contaminants is equally important. Both continuum and emission-line sources will appear in the narrow-band images. Contaminants from lower redshifts must be identified with additional data. Deep broad-band images in both the optical and near-infrared will serve this purpose. For example, for a 32hour narrow-band observation at $R=125, J$-band data that is deeper than $\sim 25.9$ (AB) should reveal all continuum sources in the narrow-band image. Many upcoming imaging surveys will exceed this sensitivity.

Extremely high redshift galaxies may be difficult to detect in the continuum, even with HST. The brightest galaxy known at $z \sim 6$ (Dickinson et al. 2003) would be detectable at $z=8.2$, without evolution, in the upcoming HST/NICMOS ultra-deep field observations. But at $z \sim 8$ galaxies should have lower star formation rates and less stellar mass. Even if the continuum flux is detectable, the target objects will be identifiable as 
z-band dropouts and distinguishable from lower redshift contaminants which will appear in the optical bands.

The remaining possible contaminants are low $-z$ objects with strong emission lines and no detectable continuum. In all cases, however, the emission lines must be prohibitively strong for the objects to remain undetected in extremely deep broad-band images. For the scenario described above, if a detected emission line in the $1.122 \mu \mathrm{m}$ window is actually $[\mathrm{O} \mathrm{II}](\lambda 3727)$, the $z=2.01$ galaxy would have to have an unphysical equivalent width of $\sim 800 \AA$ or more (rest-frame) to remain undetected in a deep F606W observation (29.3 magnitude limit at $3 \sigma$, corresponding to the HDF-North data). Other emission lines place similar requirements on the high-redshift objects, including both QSO lines and strong galaxy lines. Thus, the sources that appear in the narrow-band image but are non-detections in deep optical continuum images are almost certainly $z>7$ galaxies.

\section{GOALS FOR PRESENT AND FUTURE FACILITIES}

Many far-reaching science goals motivate devoting an enormous effort to the study of extremely high-redshift "firstlight" objects. Some of these goals are: (1) measuring the luminosity function of $z>7$ galaxies, to compare with the number of ionizing photons needed to reionize the universe, and to measure the global star formation rate at these redshifts, (2) mapping high-redshift sources and measuring their kinematics, to observe their clustering properties and compare with models of early galaxy formation, (3) quantifying high- $z$ Stromgren spheres in the IGM from Ly $\alpha$ line profiles, and (4) directly probing the IMF and metallicity of early stars with, e.g., He II $(\lambda 1640)$ and, eventually, other spectral lines.

Accomplishing these goals will require a large, complementary set of observational facilities. The James Webb Space Telescope, with broad-band sensitivities of $\sim 2 \mathrm{nJy}$, will detect early stars in their continuum light. Narrow-band imaging will likely remain the strongest tool of ground-based facilities in the near-infrared. Fig. 3 shows the expected performance of 8-meter and future 30-meter ground-based telescopes. Assuming modern detector characteristics, we plot the fluxes

\footnotetext{
${ }^{7}$ This technology is one goal of the DAZLE spectrograph, under development for the VLT (http://www.aao.gov.au/dazle/)

${ }^{8}$ At, e.g., $R=3000$, a long NIRI observation would actually be detectornoise dominated; thus, the development of low read noise and low dark cur-
}

from star-forming regions at $z=8.227$. Clearly, 8-meter class telescopes have great near-term potential for advancing the study of early star formation. Narrower filters $(R \geq 1000)$ would improve sensitivity substantially, although they would require some development in both filter and detector technology. ${ }^{7,8}$ Looking further into the future, the detailed physical study of these objects - an exploration down the luminosity function, measurements of $\operatorname{Ly} \alpha$ line profiles, and the detection of fainter spectral emission lines - is probably the role of the next generation of large ground-based telescopes. The timely discovery of high-z Ly $\alpha$ emitters is crucial to guide the design of future facilities, since probing the epoch of reionization is one of their central scientific goals.

In this Letter, we motivate the search for high-redshift galaxies by presenting plausible scenarios in which the Ly $\alpha$ emission from $z \approx 8$ galaxies is observable with 8-meter-class telescopes. The discovery of $z>7$ objects is a crucial component of the quest to understand the evolution of the early universe. They existed at an epoch when the universe was still partly neutral, and they are the best candidates for supplying the radiation that reionized the universe. Although we are able to combine many areas of recent research to arrive at an educated guess about the properties of $z>7$ objects, there are still many critical unanswered questions about the nature of reionization. With the discovery and study of $z>7$ objects, many of these questions will be answered. We stand on the verge of probing reionization directly.

We thank Margaret Geller for encouraging us to write this paper, and for reading a draft, and we thank Rob Kennicutt and Steve Furlanetto for insightful comments. Support for EJB and RAD was provided by NASA through Hubble Fellowship grants \#HST-HF-01135.01 and \#HST-HF-0128.01A, respectively, awarded by the Space Telescope Science Institute, which is operated by the Association of Universities for Research in Astronomy, Inc., for NASA, under contract NAS 5-26555.

rent detectors is another important component in the search for extremely high-redshift objects.

\section{REFERENCES}

Abel, T., Bryan, G. L., \& Norman, M. L. 2000, ApJ, 540, 39

Beasley, M. A., Kawata, D., Pearce, F. R., Forbes, D. A., \& Gibson, B. K 2003, ApJ, in press astro-ph/0309198

Becker, R. H., et al. 2001, AJ, 122, 2850

Bennett, C. L., et al. 2003, ApJ, in press astro-ph/0302207

Bertin, E., Arnouts, S. 1996, A\&AS, 117, 393

Bromm, V., Coppi, P. S., \& Larson, R. B. 1999, ApJ, 527,L5

Bromm, V., Coppi, P. S., \& Larson, R. B. 2002, ApJ, 564, 23

Bromm, V., Kudritzki, R. P., \& Loeb, A. 2001, ApJ, 552, 464

Bunker, A. J., Stanway, E. R., Ellis, R. S., McMahon, R. G., \& McCarthy, P. J. 2003, MNRAS, 342, 47

Cen, R. 2003a, ApJ, 591, 12

Cen, R. 2003b, ApJ, 591, L5-L8

Cuby, J.-G., Le Fèvre, O., McCracken, H., Cuillandre, J.-C., Magnier, E., \& Meneux, B. 2003, A\&A, 405, 19

Dickinson, M., et al. 2003, ApJ, in press astro-ph/0309070

Fan, X., et al. 2001, AJ, 122, 2833

Fan, X., et al. 2002, AJ, 123, 1247

Ferguson, H. C., Dickinson, M., \& Papovich, C. 2002, ApJ, 569, L65

Haiman, Z. 2002, ApJ, 576, L1

Hernquist, L. \& Springel, V. 2003, MNRAS, 341, 1253

Kogut, A. et al. 2003, ApJS, 148, 161

Maier, C., et al. 2003, A\&A, 402, 79

Panagia, N., Stiavelli, M., \& Fall, S. M. 2003, in Multi-Wavelength Cosmology, ed. M. Plionis (Greece: Kluwer) astro-ph/0309406
Pettini, M., Kellogg, M., Steidel, C. C., Dickinson, M., Adelberger, K., \& Giavalisco, M. 1998, ApJ, 508, 539

Rhoads, J. E., et al. 2003, AJ, 125, 1006

Santos, M. R. 2003, MNRAS, submitted astro-ph/0308196

Scannapieco, E., Schneider, R., \& Ferrara, A. 2003, ApJ, 589, 35

Schaerer, D. 2003, A\&A, 397, 527

Sokasian, A., Abel, T., Hernquist, L., \& Springel, V. 2003a, MNRAS, 334, 607

Sokasian, A., Yoshida, N., Abel, T., Hernquist, L., \& Springel, V. 2003b, MNRAS, submitted astro-ph/0307451

Spergel, D. N., et al. 2003, ApJ, in press |astro-ph/0302209|

Springel, V., \& Hernquist, L. 2002, MNRAS, 333, 649

Springel, V., \& Hernquist, L. 2003a, MNRAS, 339, 312

Springel, V., \& Hernquist, L. 2003b, MNRAS, 339, 289

Stanway, E. R., Bunker, A. J., \& McMahon, R. G. 2003, MNRAS, 342, 439

Steidel, C. C., Pettini, M., \& Adelberger, K. L. 2001, ApJ, 546, 665

Thompson, D., Djorgovski, S., Trauger, J. 1995, AJ, 110, 963

Thompson, D., Mannucci, F., \& Beckwith, S. V. W. 1996, AJ, 112, 1794

Tinsley, B. 1973, Astrophys. Lett., 14, 15

Yan, H., Windhorst, R. A., \& Cohen, S. H. 2003, ApJ, 585, L93

Yoshida, N., Sokasian, A., Hernquist, L., \& Springel, V. 2003a, ApJ, 591, L1 Yoshida, N., Sokasian, A., Hernquist, L., \& Springel, V. 2003b, ApJ, in press astro-ph/0305517 